\title{
A PRIMARILY CLINICIAN'S RESPONSIBILITY
}

\author{
Momcilo Jankovic $^{1}$, Andrea Biondi ${ }^{2}$, Francesca Gallina ${ }^{3}$, and Diego Cortinovis ${ }^{3}$ \\ ${ }^{1}$ Maria Letizia Verga Center, Department of Paediatrics, University of Milano-Bicocca, \\ ASST Monza/MBBM Foundation, Monza \\ ${ }^{2}$ Ospedale S. Gerardo, University of Milano-Bicocca, Fondazione MBBM, Monza, Italy \\ ${ }^{3}$ San Gerardo Hospital
}

January 25, 2022

\section{A PRIMARILY CLINICIAN'S RESPONSIBILITY}

\author{
MomciloJankovic, MD; Dept. of Pediatrics, Univ. Milano-Bicocca / Found. MBBM
}

Andrea Biondi, MD; University of Milano-Bicocca

Francesca Gallina, PhD; San Gerardo Hospital, MedicalOncology

Diego Cortinovis MD; San Gerardo Hospital, MedicalOncology

We are a team ofItalian hemato oncologist pediatricians, adult oncologists, and psychologists that,for quite some time, havebeen involved in clinical research on communication strategies between cancer patients and their children.

Based on our experience, we strongly feel that it is an ethical duty for all clinicians to make children awareof what is happening to one of their parents when they are diagnosed with cancer. Thus, in our opinion, the issue is not whether to talk with the children about their parent's illness or not, but rather how to do that in a tactful and effective manner. To this end, we carried out a series ofparent-child dialogue-based interventionsthrough a multidisciplinary approach. The successful outcome of our interventions was measured and validated through qualitative assessment of a self-reported questionnaire administered to parents and children - even shortly after the death of the ill parent.

The lesson we have learned from this 20-year-long survey is that listening to what the children have to sayabout their hopes, fears and worriesas well as answering to their many questions is crucial tomake them understand and accept the unfortunate event that has happened toone of their parents. In our experience, it is up to the clinician to build a relationship of mutual trust with the child - it is primarily the clinician's responsibility.

This interventionalso requires the presence of a psychologist - but not that of any of the two parents - that will offer guidance toall family members throughout the illness trajectory. Once the psychologistagrees to undertake this task, he/shehasto strongly believe in it, and this will give him/her the strength needed to face the hardship of this journey. The two other members of the multidisciplinary team (MDT), a pediatrician and an oncologist,are not just supposed toensure the best medical care. They should guarantee the psychophysical well-being of the entire family unit, including the children.

In the end - we cannot deny it-, we feel empty inside but,at the same time, happy to have contributed to tackling such a challenging issue, which is still a taboo that we need to break by making it an integral part of our healthcare system. 
Let's not forget that "the moon" (we, the healthcare providers) and "the stars" (all the people who dedicate time and resources to the patient) work together to light upthe "dark" path (the disease) of a child or an adult. As clinicians,our main goal is to improve the quality of lifeof all family members we assist- those who recover and those who die - before, during, and after the disease course, regardless of the outcome.

Elizabeth Kubler-Ross wrote: "People are like stained-glass windows. They sparkle and shine when the sun is out, but when the darkness sets in, their true beauty is revealed only if there is a light from within." ... and even children have this inner light. Let's help them keep it lit even when facing the pain caused by the death of one of their parents. 